CELL BIOLOGY OF THE NEURON

\title{
FMRP and its many partners
}

Two studies, published in Cell and Neuron, give new insight into the possible functions of the protein FMRP. Mutations in the gene Fmr1, which encodes FMRP, cause fragile X syndrome, the most common cause of mental retardation. FMRP binds to various mRNAs, but its normal role and how disruption of that function causes the syndrome are unclear.

Myashiro et al. used an approach that allowed them to identify the RNAs that are associated with FMRP in vivo. The technique, called antibody-positioned RNA amplification (APRA), uses a monoclonal antibody to position a priming/amplification oligonucleotide close to mRNAs in the FMRP complex, leading to cDNA synthesis. The cDNA, complexed with the antibody, can subsequently be removed from the cell and amplified in vitro for identification by screening against microarrays.

Using this technique, and screening against two arrays, the authors identified more than 1,000 putative in vivo targets of FMRP binding. They then used more traditional methods - filter-binding assays and ultraviolet crosslinking - to confirm that around $60 \%$ of the mRNAs could interact directly with FMRP. Many of these mRNAs encoded proteins that are important for synaptic plasticity and neuronal development.

Myashiro et al. hypothesized that the interaction between these mRNAs and FMRP was important for regulating the distribution or abundance of proteins in neurons. They therefore compared control mice with those lacking Fmr1, and found that a subset of the mRNAs showed altered abundance and subcellular distribution in neural tissue in the Fmr1 null mice. The proteins encoded by these mRNAs were also distributed differently.

In the second study, Zalfa et al. compared the translational efficiency of various mRNAs in wild-type and Fmr1-null mice. They found that a subset of mRNAs were more efficiently translated in the knockout mice, and that the effect was most marked in synaptoneurosomal preparations. This indicates that FMRP might suppress the translation of specific mRNAs at synapses.

The authors also discovered that FMRP binds to a non-translated RNA, long known to reside at synapses, called $B C 1$, and that blocking $B C 1$ interferes with the ability of FMRP to associate with its target mRNAs. $B C 1$ can also interact directly with the mRNAs that are regulated by FMRP. Zalfa et al. suggest that $B C 1$ might be responsible for targeting FMRP to its target mRNAs.

Both of these studies identify important mRNAs, the subcellular localization and translation of which seem to be regulated by FMRP, particularly at synapses. This clearly points towards a potential mechanism for the various effects of fragile $\mathrm{X}$ syndrome, showing how a mutation in just one gene could disrupt many vital pathways in the nervous system.

Rachel Jones

(D) References and links ORIGINAL RESEARCH PAPERS Zalfa, F. et al. ORIGINAL RESEARCH PAPERS Zalfa, F. et al.
The fragile X syndrome protein FMRP associates with BC1 RNA and regulates the transmission of specific mRNAs at synapses. Cell 112, 317-327 (2003) | Miyashiro, K. Y. et al. RNA cargoes associating with FMRP reveal deficits in cellular functioning in $F m r 1$ null mice. Neuron $\mathbf{3 7}$, functioning in $F m / 1$
$417-431$
(2003)

FURTHER READing Chelly, J. \& Mandel, J.-L. Monogenic causes of $X$-linked mental retardation. Nature Rev. Genet. 2, 669-680 (2001)

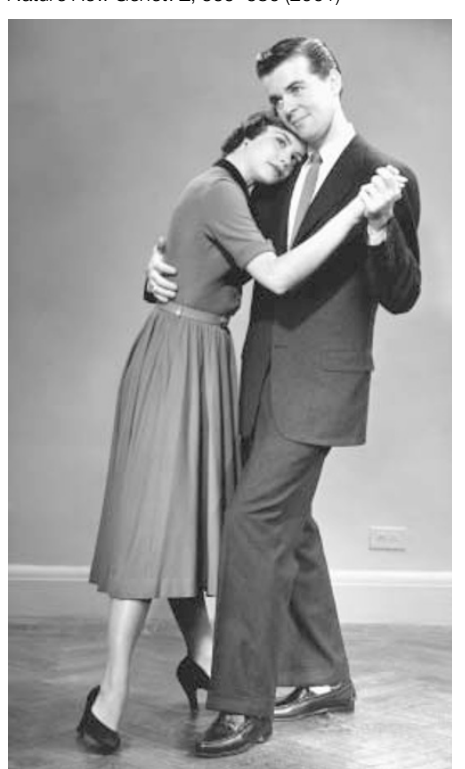

NEURAL NETWORKS

\section{Making waves}

Neuronal assemblies in the hippocampus are central to the encoding, consolidation and retrieval of memories. The activity patterns of networks of hippocampal neurons change in association with different behaviours, and these distinct network states

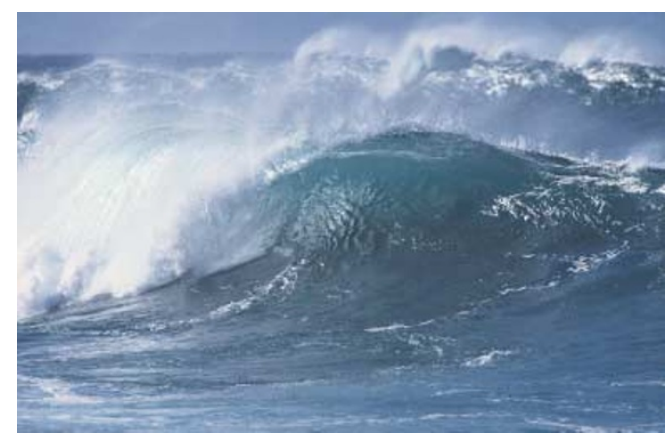
are proposed to contribute to specific memory processes. Inhibitory interneurons are known to synchronize pyramidal cell firing in the hippocampus. In a paper published in Nature, Klausberger and colleagues now provide evidence that the diversity of interneurons helps to shape the brain-state-dependent activity of hippocampal neural networks.

The hippocampus houses an assortment of interneurons, which can be classified on the basis of several properties, including their specific innervation of distinct domains of pyramidal cells. Klausberger et al. examined the possibility that different interneuron classes contribute differentially to network activity patterns. They made electrophysiological recordings in the CA1 region of anaesthetized rats from three types of interneuron - basket, axo-axonic and oriens-lacunosum-moleculare (O-LM) cells - which were visualized and distinguished by their patterns of synaptic connectivity and by the use of neurochemical markers. The firing patterns of these interneurons were monitored during two types of network activity: theta oscillations $(4-8 \mathrm{~Hz})$, which are associated with exploratory behaviour and rapid-eyemovement sleep in non-anaesthetized animals, and sharp-waveassociated ripple oscillations $(120-200 \mathrm{~Hz})$, which are observed during periods of inactivity and in slow-wave sleep.

The authors found that, whereas firing patterns generated by single cells of the same class were remarkably alike, serving as a unique 'signature' for each type of interneuron, the phase preferences of these classes during theta or ripple activity were markedly different. For example, during theta oscillations, basket cells fired preferentially on the descending phase of each wave, axo-axonic cells fired right after the peak of the theta wave, and $\mathrm{O}-\mathrm{LM}$ cells fired rhythmically at the trough of the theta cycle. It seems likely that these three types of interneuron make specific contributions to the production of network oscillations through their characteristic patterns of activity.

Further studies are warranted to find out exactly how the precisely timed firing of these interneurons coordinates the activity of hippocampal pyramidal cells, and to extend these observations to other interneuron classes, to other network states and, importantly, to non-anaesthetized, behaving animals.

Rebecca Craven, Senior Subeditor, Nature

(2) References and links

ORIGINAL RESEARCH PAPER Klausberger, T. et al. Brain-state- and cell-type-specific

firing of hippocampal interneurons in vivo. Nature 421, 844-848 (2003)

FURTHER READING McBain, C. J. \& Fisahn, A. Interneurons unbound. Nature Rev.

FURTHER READING MCB
Neurosci. 2, 11-23 (2001) 